\title{
Environmental Chamber for In Situ Studying of Drying of Granular Materials Using X-ray Computer Tomography Microscopy
}

\author{
M.M. Sushchikh, ${ }^{*}$ S.Yu. Sushchikh, ${ }^{*}$ and T.G. Theofanous* \\ * Center for Risk Studies and Safety, University of California at Santa Barbara, Santa Barbara, CA, \\ 93106-1070
}

Evolution of limited amounts of liquids in granular materials is a subject of interest because of environmental and industrial needs. Examples are water transport is soils [1], fate of contaminants [2] and processing of granular materials [3]. Understanding of these processes requires detailed and microscopic knowledge of the matrix geometries and liquid patterns during "spreading" and depletion over time. X-ray computer tomography (XCT) microscopy is probably the only technique which is capable of producing 3D images of real materials with micron-scale resolution and now it is reasonably affordable. Existing instruments are designed for the "dead" and bare samples imaged at the instrument bench and this limits the utility when internal mass distributions are non-static on the time period of hours needed for data acquisition in XCT. In this presentation we show how this issue can be addressed by suppressing the evaporation for time periods of CT scans (several hours each), while allowing normal evolution under well controlled conditions of pressure, temperature and carrier gas flow on the time scale of weeks or even months.

The so-designed and built prototype of an Environmental Chamber (EC) is illustrated in Fig.1. The chamber was design to fit inside MicroXCT-200 instrument (Xradia Inc, Concord, CA). The chamber body does not rotate during the scan thus the absorption of the X-rays by the chamber walls remains fixed and is precisely negated in the CT without deteriorating the image of the sample. The rotation of the sample held in the centre of the chamber is driven by the rotation of the base on which the chamber rests - on near frictionless bearings. A carrier gas can be introduced at the chamber top trough multiple small holes and it exits through two large openings at the bottom. The gas flow geometry is designed with the help of FLUENT CFD software (ANSYS, Inc., Canonsburg, PA) so as to obtain a well defined diffusion boundary layer as needed for mass loss calculations, while avoiding creation of any stagnation areas (Fig. 1, right). The chamber has an insertion port for a capillary which allows sampling of the outgoing gas/vapor mixture by a residual gas analyzer. All lines needed to the outside are routed through existing feed through so that shielding is not compromised.

The evolution of liquids in granular materials is special in that the particles in the granular materials can move relative to each other. When a liquid introduced, the capillary forces become the decisive factor for formation of a new geometry and further evolutions like spreading and drying are accompanied by changes in the particle positions. Thus the evolution of both, the liquid and the matrix must be recorded in order to characterize the process comprehensively. Obviously the movements of the solid particles due to external factors such as vibrations of the gas flow forces are to be avoided. We have verified the stability of the positions of the glass balls held in the glass tube in the center of the chamber by recording a series of the MicroXCT 3D images in a stagnant nitrogen atmosphere while between the measurements the $100 \mathrm{SCCM} \mathrm{N}_{2}$ flow was kept through the chamber for an hour. Figure 2 shows the horizontal cross-section images of the most fragile top layer of the glass balls which are taken from three different runs. The spheres did not move between the runs. 
Thus we have designed, built and tested a new accessory for the Xradia MicroXCT-200 instrument. This accessory allows in situ studying of the evolution of liquids in granular and porous materials by providing controlled environment [4].

References

[1] L. Mahrt and H. Pan, Bound.-Layer Meteor., 29 (1984).

[2] R.D. Wauchope, J. Environ. Qual., 7 (1978).

[3] I. Talu, G.I. Tardos, and M.I. Khan, Powder Technol., 110 (2000).

[4] Work supported by the Defense Threat Reduction Agency of the US Department of Defense.
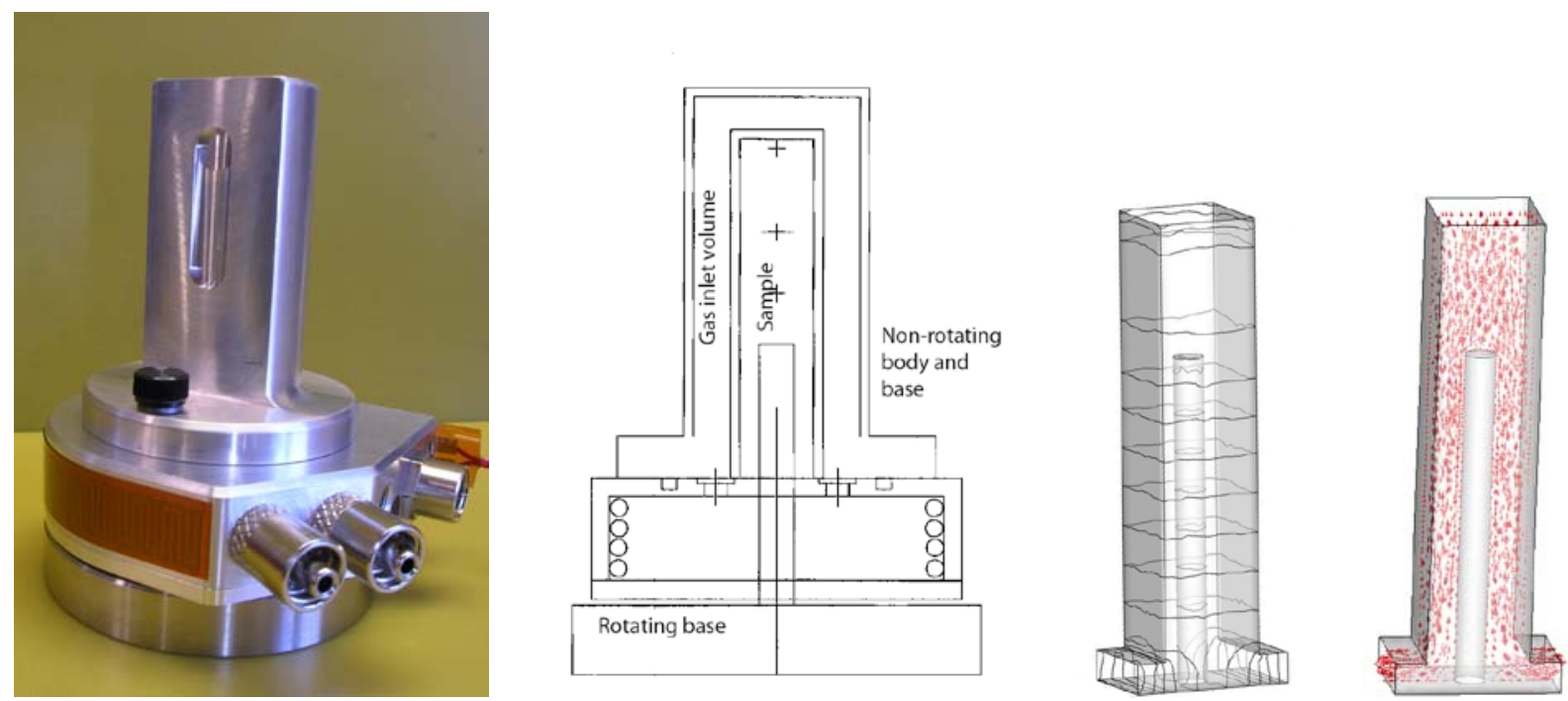

FIG. 1. The environmental chamber for keeping a sample at controlled environment during X-ray CT scan (left). Calculated gas velocity field inside the chamber (right). Nitrogen at 100 SCCM flow rate.

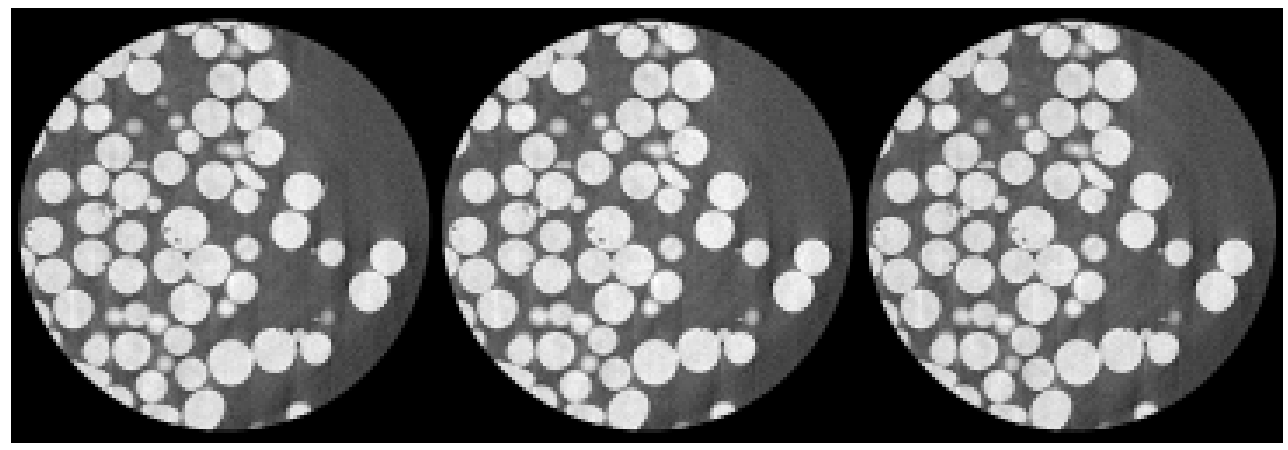

FIG. 2. Horizontal cross-sections of a top layer of glass balls (150-212 $\mu \mathrm{m}$ diameter) extracted from MicroXCT 3D images obtained in three separate scans. Identity of the images proves that the balls did not move during a total of 40 hours of operation of the instrument. 